\title{
Dietary fat modifies lipid metabolism in the adipose tissue of metabolic syndrome patients
}

\author{
Antonio Camargo • María E. Meneses · Pablo Pérez-Martínez · Javier Delgado-Lista \\ Oriol A. Rangel-Zúñiga · Carmen Marín · Yolanda Almadén · Elena M. Yubero-Serrano • \\ Lorena González-Guardia · Francisco Fuentes · Francisco J. Tinahones • Helen M. Roche • \\ María M. Malagón · Francisco Pérez-Jiménez · José López-Miranda
}

Received: 14 February 2014/ Accepted: 13 May 2014/Published online: 4 June 2014

(C) Springer-Verlag Berlin Heidelberg 2014

\begin{abstract}
Adipose tissue (AT) is a key organ in the regulation of total body lipid homeostasis, which is responsible for the storage and release of fatty acids according to metabolic needs. We aimed to investigate the effect of the quantity and quality of dietary fat on the lipogenesis and lipolysis processes in the AT of metabolic syndrome (MetS) patients. A randomized, controlled trial conducted within the LIPGENE study assigned MetS patients to one of four diets: (a) high-saturated fatty acid (HSFA) (b) highmonounsaturated fatty acid, and (c, d) two low-fat, highcomplex carbohydrate diets supplemented with long chain (LC) n-3 (LFHCC n-3) polyunsaturated fatty acids (PUFA) or placebo (LFHCC), for 12 weeks each. A fat challenge reflecting the same fatty acid composition as the original
\end{abstract}

Clinical Trial Registration Number: ClinicalTrials.gov

NCT00429195. The experimental protocol was approved by the local Ethics Committee, in line with the Helsinki Declaration.

Electronic supplementary material The online version of this article (doi:10.1007/s12263-014-0409-3) contains supplementary material, which is available to authorized users.

A. Camargo - M. E. Meneses - P. Pérez-Martínez .

J. Delgado-Lista $\cdot$ O. A. Rangel-Zúñiga $\cdot$ C. Marín ·

Y. Almadén · E. M. Yubero-Serrano · L. González-Guardia ·

F. Fuentes · F. Pérez-Jiménez · J. López-Miranda $(\bowtie)$

Lipids and Atherosclerosis Unit, IMIBIC/Reina Sofia University

Hospital, University of Cordoba, Avda. Menendez Pidal, s/n.,

14004 Córdoba, Spain

e-mail: jlopezmir@uco.es

A. Camargo - M. E. Meneses - P. Pérez-Martínez .

J. Delgado-Lista $\cdot$ O. A. Rangel-Zúñiga $\cdot$ C. Marín ·

Y. Almadén · E. M. Yubero-Serrano · L. González-Guardia ·

F. Fuentes · F. J. Tinahones - M. M. Malagón ·

F. Pérez-Jiménez · J. López-Miranda

CIBER Fisiopatología de la Obesidad y Nutrición (CIBEROBN),

Instituto de Salud Carlos III, Madrid, Spain diets was conducted post-intervention. Long-term consumption of the LFHCC diet induced an increase in the fasting expression levels of the sterol regulatory element binding protein-1 and stearoyl-CoA desaturase D9-desaturase genes, whereas the supplementation of this diet with n-3 PUFA reversed this effect $(p=0.007)$. In contrast, long-term consumption of the HSFA diet increased the expression of the adipose triglyceride lipase (ATGL) gene, at both fasting and postprandial states (both, $p<0.001$ ). Our results showed the anti-lipogenic effect exerted by LC n-3 PUFA when administered together with a LFHCC diet. Conversely, a diet high in saturated fat increased the expression of the lipolytic gene ATGL relative to the other diets.

Keywords Adipose tissue - Diet - Lipolysis · Lipogenesis $\cdot$ Metabolic syndrome

\author{
F. J. Tinahones \\ Endocrinology and Nutrition Service, Hospital Virgen de la \\ Victoria, Málaga, Spain \\ H. M. Roche \\ UCD Institute of Food and Health/UCD Conway Institute, \\ School of Public Health and Population Sciences, University \\ College Dublin, Dublin, Ireland \\ M. M. Malagón \\ Department of Cell Biology, Physiology, and Immunology, \\ IMIBIC/Reina Sofia University Hospital, University of Córdoba, \\ Cordoba, Spain
}




\section{Introduction}

Adipose tissue (AT) is an important organ for whole-body lipid homeostasis, metabolically flexible, and readily able to switch from a fatty acid-storing to a fatty acid-releasing function, according to the metabolic needs. Moreover, excess of nutrients causes an increase in the size (hypertrophy) and number (hyperplasia) of adipocytes during the development of obesity (Sun et al. 2011). However, the ability of AT to store excess energy as triglycerides is limited, as determined by both genetic and environmental factors. In fact, it has been proposed that when the lipid storage capacity of AT is exceeded, then lipids accumulate ectopically in the liver and muscle, causing insulin resistance and impaired pancreatic beta cell function by a lipotoxic mechanism (Suganami et al. 2012).

The AT triglyceride content depends primarily on the balance between lipogenesis and lipolysis, two opposing processes regulated by a complex interaction of several factors including circulating hormones, such as insulin, as well as by adipose-derived factors, such as leptin and adiponectin, which in turn have an important role on insulin action (Havel 2002).

It has been shown that obese and metabolic syndrome (MetS) patients exhibit altered lipogenic and lipolytic processes (Cifuentes et al. 2008). It has also been shown that the transcription factor sterol regulatory element binding protein-1 (SREBP1), which activates the expression of several genes involved in fatty acid synthesis, is overexpressed in animal models of insulin resistance and obesity (Shimomura et al. 2000). It has been suggested that the increased expression of lipogenic genes in adipocytes leading to fat accretion and adipocyte hypertrophy exerts an antagonistic effect on insulin action, due to substrate competition with glucose or to a lipotoxic effect, or both (Schmitz-Peiffer 2000), which finally contribute to the development of type 2 diabetes mellitus (T2DM) (Weyer et al. 2000).

However, to the best of our knowledge, the effect of dietary fat on the expression of genes related to lipid metabolism in the AT has not been reported previously in humans, either during fasting or in the postprandial state.

The latter is particularly relevant because most of the hours in the day are typically spent in the fed state, and abnormalities in lipid metabolism in AT could contribute with a pro-atherogenic state, especially in MetS patients, who are vulnerable since MetS patients show an exacerbated hypertriglyceridemia response (Khoury et al. 2009).

The aim of this study was to clarify how the quantity and quality of dietary fat influence the regulation of lipid metabolism in fasting and in the postprandial state by assessing the expression of key genes involved in lipolysis and lipogenesis in AT in MetS patients.

\section{Methods and procedures}

Participants and recruitment

This study was carried out in the Lipids and Atherosclerosis Unit at the Reina Sofia University Hospital, from February 2005 to April 2006 within the framework of the LIPGENE study (Diet, genomics and metabolic syndrome: an integrated nutrition, agro-food, social and economic analysis), a Framework 6 Integrated Project funded by the European Union. A subgroup of 39 patients with MetS (HSFA: $3 / 5$ (men/women); HMUFA: 3/6; LFHCC: 4/8; LFHCC n-3: 4/6) from the Spanish cohort of the multicenter LIPGENE study (Clinical Trial Registration Number: NCT00429195) successfully concluded the dietary intervention and the postprandial study post-intervention. All participants gave written informed consent and underwent a comprehensive medical history, physical examination, and clinical chemistry analysis before enrollment. The experimental protocol was approved by the local ethic committee according to the Helsinki Declaration.

Study design

Metabolic syndrome (MetS) patients, according to published criteria (Grundy et al. 2004), were randomly stratified to 1 of 4 dietary interventions (isoenergetic diets) for 12 week, which conformed to the LIPGENE inclusion and exclusion criteria (Shaw et al. 2009). Post-intervention, a fat challenge (test meal) was administered providing the same amount of fat $(0.7 \mathrm{~g} / \mathrm{kg}$ body weight), wherein the fatty acid composition reflected that consumed within the intervention period. The intervention study design and intervention protocol, which also provides information about food consumption and dietary compliance have been described in detail by Shaw et al. (2009). Briefly, dietary intake and compliance were assessed by a 3-day (2 weekdays and 1 weekend day) weighed food intake assessments at baseline, week 6, and week 12. Dietary analysis program reflective of the food choices were used (Dietsource version 2.0).

\section{Randomization and intervention}

Randomization and intervention have been previously described in detail (Meneses et al. 2011; Shaw et al. 2009). Briefly, randomization was completed according to age, gender, and fasting plasma glucose concentration. Two diets were designed to provide $38 \%$ energy (E) from fat: a high-fat, saturated fatty acid-rich diet (HSFA), which was designed to provide $16 \% \mathrm{E}$ as SFA, and a high-monounsaturated fatty acid-rich diet (HMUFA) designed to provide $20 \% \mathrm{E}$ from MUFA. The other two diets were low-fat, 
high-complex carbohydrates-rich diet (LFHCC and LFHCC (n-3); $28 \%$ E from fat); the LFHCC (n-3) diet included a 1.24-g/day supplement of long chain (n-3) PUFA [ratio of 1.4 eicosapentaenoic acid (EPA): 1 docosahexaenoic acid (DHA)], and the LFHCC diet included a 1.2-g/day supplement of control high-oleic sunflower seed oil capsules (placebo). Fat challenge was administered to the patients at post-intervention (at week 12) in the clinical intervention center at 8:00 a.m. following a 12-h fasting and refrained from smoking during the fasting period and abstained from alcohol intake during the preceding 7 days. Composition of diets and dietary targets, as well as the composition of the test meals has been previously described (Meneses et al. 2011). Test meals provided an equal amount of fat $(0.7 \mathrm{~g} / \mathrm{kg}$ body weight $), \mathrm{E}$ content $(40.2 \mathrm{~kJ} /$ $\mathrm{kg}$ body weight), cholesterol ( $5 \mathrm{mg} / \mathrm{kg}$ of body weight), fiber, and vitamin A [62.9 mmol vitamin A (retinol) $/ \mathrm{m}^{2}$ body surface area]. The test meal provided $65 \%$ of $\mathrm{E}$ as fat, $10 \%$ as protein, and $25 \%$ as carbohydrates. During the postprandial assessment, participants rested, and did not consume any other food, but were allowed to drink water.

Blood measurements, subcutaneous adipose tissue samples collection, and RNA isolation

Blood measurements and subcutaneous adipose tissue samples were obtained, and the RNA was isolated as previously described (Meneses et al. 2011). In short, blood was collected in tubes containing EDTA, and plasma separated from red cells by centrifugation. Analytes were determined centrally by laboratory investigators of the Lipid and Atherosclerosis Unit at the Reina Sofia University Hospital. Adipose tissue samples were collected from the superficial abdominal subcutaneous adipose in the fasting state both at pre-intervention and post-intervention, and $4 \mathrm{~h}$ after the administration of the fat challenge. Adipose tissue samples were homogenized using an UltraTurrax T25 homogenizer (IKA Labortechnik), and the RNA isolated using the commercial kit RiboPure (Ambion, Applied Biosystem, Austin, TX, USA).

Quantitative RT-PCR analysis

RT-PCR was performed using the commercial kit MessageBOOSTER cDNA Synthesis Kit for qPCR (Epicentre, Madison, WI, USA), according to the manufacturers instructions and PCR analyses performed using the OpenArray $^{\mathrm{TM}}$ NT Cycler system (Applied Biosystems, Carlsbad, CA, USA), according to the manufacturer's instructions. Primer pairs were selected from the database TaqMan Gene Expression assays (Applied Biosystems, Carlsbad, CA, USA) https://products.appliedbiosystems.
com/ab/en/US/adirect/ab?cmd=catNavigate $2 \&$ catID $=$ 601267, for the following genes: sterol regulatory element binding protein 1 (SREBP1, Hs01088691_ml), stearoylCoA desaturase D9-desaturase (SCD1), fatty acid synthase (FASN), fatty acid desaturase 1 (FADS1, Hs00203685_ml), fatty acid desaturase $2\left(F A D S 2, H s 00188654 \_m l\right)$, fatty acid desaturase 3 (FADS3, Hs00222230_ml), dihydroceramide desaturase 1 (DEGSl, Hs00186447_ml), dihydroceramide desaturase 2 (DEGS2, Hs01380343_ml), adipose triglyceride lipase (ATGL, Hs00386101_ml), lipase, hormone-sensitive (HSL, Hs00943410_ml), and monoglyceride lipase (MGL, Hs00200752_ml). The relative expression of each gene was calculated using the ribosomal protein, large, P0 ( $\left.R P L P 0, H s 99999902 \_m l\right)$ as housekeeping gene. The data set was analyzed by OpenArray $^{\circledR}$ Real-Time qPCR Analysis Software (Applied Biosystems, Carlsbad, CA, USA).

Statistical analyses

Statistical analysis was performed using PASW Statistics, Version 18 (Chicago, IL, USA). The normal distribution of variables to characterize the postprandial response was assessed using the Kolmogorov-Smirnov test. We performed One-way ANOVA statistical analysis to determine the effect of the dietary intervention in fasting and the postprandial state, with diet as the inter-subject factor. Post hoc statistical analysis was completed by using the Bonferroni's multiple comparison tests. A probability of $<0.05$ was considered significant. A study of the relationship among parameters was also carried out using Pearson's linear correlation coefficient. All data presented are expressed as mean \pm SEM.

\section{Results}

\section{Baseline characteristics}

No significant differences were observed in the baseline characteristics of the 39 subjects with MetS participating in the dietary intervention (Supplemental Table 1).

Diet and lipogenesis in adipose tissue

We analyzed the AT expression of several genes regulating lipogenesis in adipocytes (SREBP1, FASN, SCDI) (Fig. 1). This showed that the gene expression of SREBPl is modulated by dietary fat $(p=0.010)$. A post hoc Bonferroni's multiple comparison test between the groups showed a statistically significant relative increase in SREBPI mRNA levels after long-term consumption of the LFHCC diet, as compared to the consumption of the LFHCC n-3 diet, 


\section{SREBP-1}

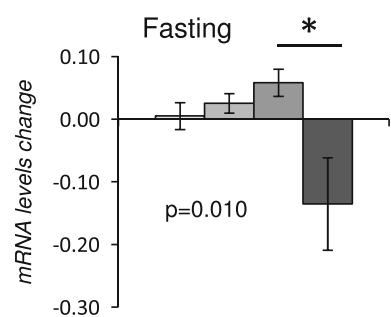

口HSFA $\square$ HMUFA $\square$ LFHCC $\square$ LFHCC n-3

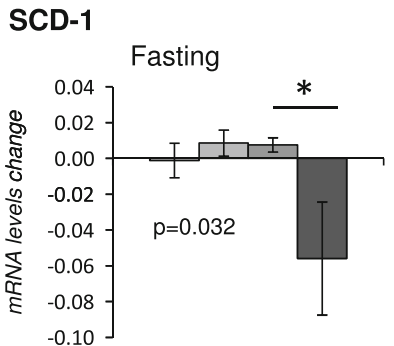

口HSFA $\square$ HMUFA $\square$ LFHCC $\square$ LFHCC $n-3$

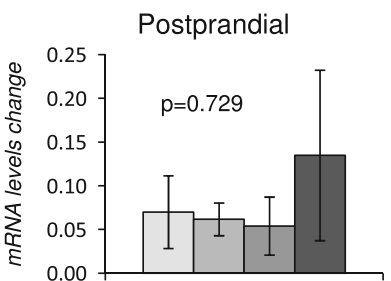

口HSFA $\square$ HMUFA $\square$ LFHCC $\square$ LFHCC $n-3$

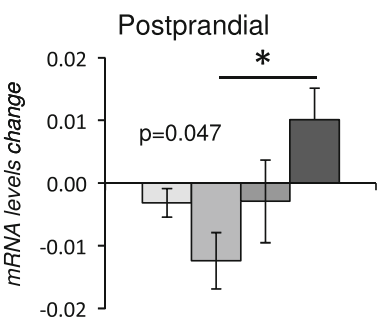

口HSFA $\square$ HMUFA $\square$ LFHCC $\square$ LFHCC $n-3$

Fig. 1 Effect of diet on the gene expression of lipogenesis-related genes. Values are the mean $( \pm \mathrm{SEM})$ of the difference in AT mRNA levels in 39 MetS patients. Fasting: changes in the mRNA levels between fasting state post-intervention and fasting state pre-intervention. Postprandial: changes in the mRNA levels between postprandial states, $4 \mathrm{~h}$ after a fat challenge reflecting the fatty acid composition of the ended diet administered post-intervention and fasting state postintervention. HSFA high-saturated fatty acid-rich diet, group with 3 men/5 women; HMUFA high-monounsaturated fatty acid-rich diet, group with 3 men/6 women, LFHCC low-fat, high-complex carbohydrate diets supplemented with placebo, group with $4 \mathrm{men} / 8$ women, LFHCC n-3 low-fat, high-complex carbohydrate diets supplemented with $\mathrm{n}-3$ polyunsaturated fatty acids (PUFA), group with $4 \mathrm{men} / 6$ women. One-way ANOVA statistical analysis between diets. Asterisk means $p<0.05$ in Post Hoc Bonferroni's multiple comparison tests

which produced SREBPI mRNA levels similar to those observed before the intervention period $(p=0.007)$. We also observed that the long-term consumption of diets differing in fat quantity and quality modulates the mRNA levels of $S C D 1$ in AT $(p=0.032)$. Specifically, SCD1 mRNA levels decreased after consumption of the LFHCC $\mathrm{n}-3$ diet, relative to the increase observed after LFHCC diet $(p=0.043)$. We were unable to detect FASN mRNA expression under our experimental conditions.

We did not observe any statistically significant changes in the SREBPI mRNA levels in the postprandial state. However, we observed a postprandial effect in SCDI mRNA levels after the intake of the four different meals $(p=0.047)$. Thus, we observed a relative increase in the LFHCC n-3 SCD1 gene expression as compared to the reduction in the $S C D 1$ transcript after consumption of a HMUFA meal $(p=0.029)$. In a similar way to what was observed in the fasting state, FASN transcripts were not detected in AT in the postprandial period.

\section{FADS-3}

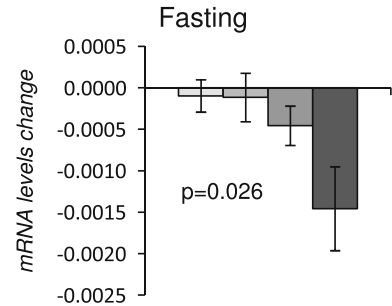

$\square$ HSFA $\square$ HMUFA $\square$ LFHCC $\square$ LFHCC $n-3$

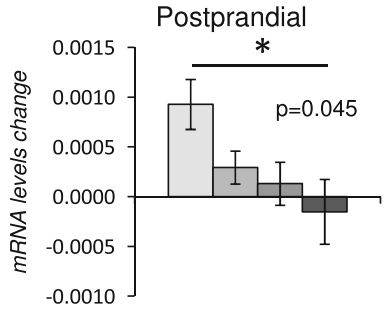

$\square$ HSFA $\square$ HMUFA $\square$ LFHCC $\square$ LFHCC $n-3$
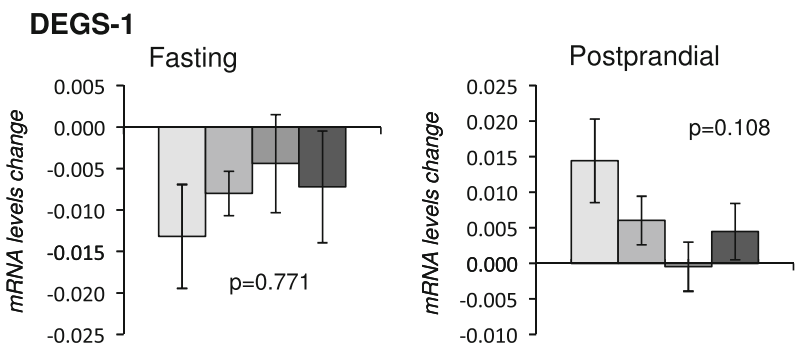

$\square$ HSFA $\square$ HMUFA $\square$ LFHCC $\square$ LFHCC $n-3$

$\square$ HSFA $\square$ HMUFA $\square$ LFHCC $\square$ LFHCC $n-3$

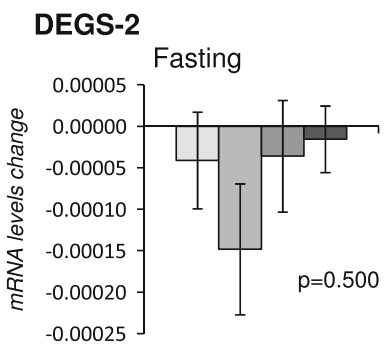

口HSFA $\square$ HMUFA $\square$ LFHCC $\square$ LFHCC $n-3$

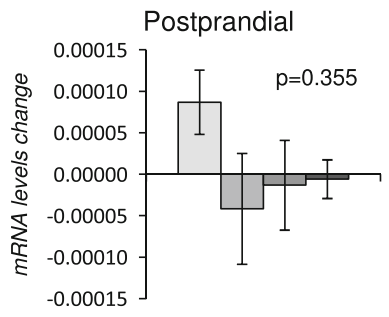

口HSFA $\square$ HMUFA $\square$ LFHCC $\square$ LFHCC $n-3$
Fig. 2 Effect of diet on the gene expression of desaturases encoding genes. Values are the mean $( \pm$ SEM) of the difference in AT mRNA levels in 39 MetS patients. Fasting: changes in the mRNA levels between fasting state post-intervention and fasting state pre-intervention. Postprandial: changes in the mRNA levels between postprandial states, $4 \mathrm{~h}$ after a fat challenge reflecting the fatty acid composition of the ended diet administered post-intervention and fasting state postintervention. HSFA high-saturated fatty acid-rich diet, group with 3 men/5 women, HMUFA high-monounsaturated fatty acid-rich diet, group with 3 men/6 women; LFHCC low-fat, high-complex carbohydrate diets supplemented with placebo, group with 4 men/8 women; LFHCC n-3 low-fat, high-complex carbohydrate diets supplemented with n-3 polyunsaturated fatty acids (PUFA), group with 4 men/6 women. One-way ANOVA statistical analysis between diets. Asterisk denotes $p<0.05$ in Post Hoc Bonferroni's multiple comparison tests

Diet and fatty acid desaturation in adipose tissue

We also studied the expression of the fatty acid desaturase (FADS) gene family members FADS1 and FADS2 (Fig. 2), which encode for $\Delta 5$ - and $\Delta 6$-fatty acid desaturase activities (involved in polyunsaturated fatty acid biosynthesis), respectively, and FADS3, whose function has not been established yet (Pedrono et al. 2010). Although the mRNA levels for FADS1 and FADS2 were not detected under our experimental conditions, we observed a relative decrease in 


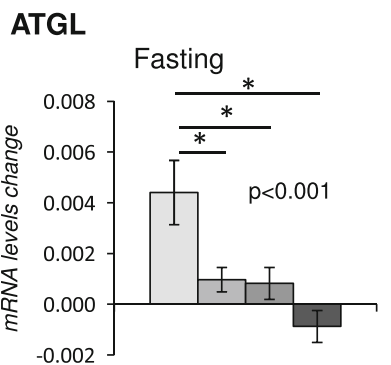

$\square$ HSFA $\square$ HMUFA $\square$ LFHCC $\square$ LFHCC n-3

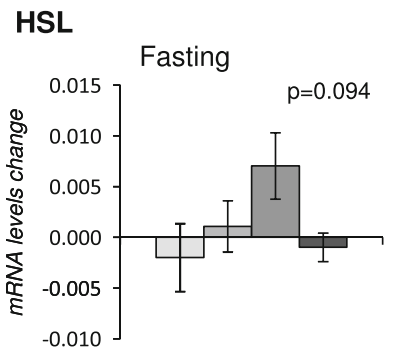

$\square$ HSFA $\square$ HMUFA $\square$ LFHCC $\square$ LFHCC n-3

MGL

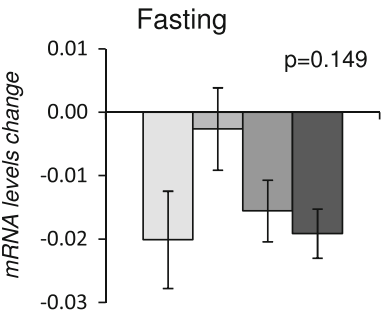

口HSFA $\square$ HMUFA $\square$ LFHCC $\square$ LFHCC $n-3$

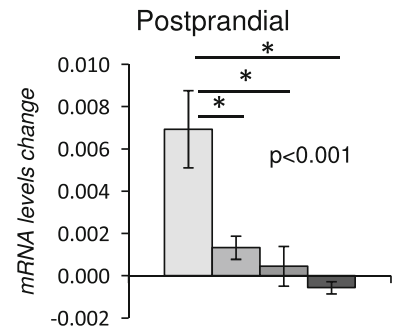

$\square$ HSFA $\square$ HMUFA $\square$ LFHCC $\square$ LFHCC $n-3$

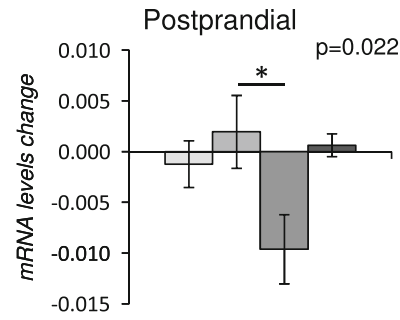

口HSFA $\square$ HMUFA $\square$ LFHCC $\square$ LFHCC $n-3$

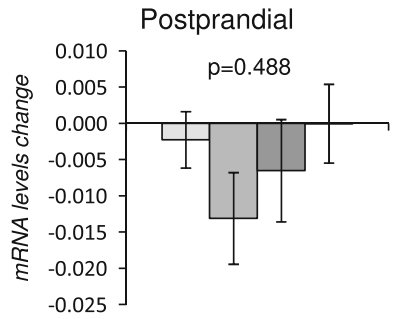

$\square$ HSFA $\square$ HMUFA $\square$ LFHCC $\square$ LFHCC $n-3$

Fig. 3 Effect of diet on the gene expression of lipolysis-related genes. Values are the mean $( \pm \mathrm{SEM})$ of the difference in AT mRNA levels in 39 MetS patients. Fasting: changes in the mRNA levels between fasting state post-intervention and fasting state pre-intervention. Postprandial: changes in the mRNA levels between postprandial states, $4 \mathrm{~h}$ after a fat challenge reflecting the fatty acid composition of the ended diet administered post-intervention and fasting state postintervention. HSFA high-saturated fatty acid-rich diet, group with 3 men/5 women, HMUFA high-monounsaturated fatty acid-rich diet, group with 3 men/6 women, LFHCC low-fat, high-complex carbohydrate diets supplemented with placebo, group with 4 men/8 women; LFHCC $n$-3 low-fat, high-complex carbohydrate diets supplemented with $n-3$ polyunsaturated fatty acids (PUFA), group with 4 men/6 women. One-way ANOVA statistical analysis between diets. Asterisk denotes $p<0.05$ in Post Hoc Bonferroni's multiple comparison tests

the gene expression of FADS3 as the unsaturation grade of the fat in the diet increased. In fact, post hoc Bonferroni's multiple comparison tests showed a postprandial increase in the expression of FADS 3 after HSFA diet consumption relative to the LFHCC n-3 diet.

Additionally, we quantified the expression of the two dihydroceramide desaturases, DEGS1 and DEGS2 (Fig. 2), which are responsible for converting dihydroceramide into

ceramide in the de novo sphingolipid biosynthesis pathway (Breen et al. 2013; Ternes et al. 2002). Although we observed a trend toward a postprandial increase in the expression of these genes with the HSFA meal relative to the other meals and a decrease in parallel with the unsaturation grade of the fat ingested, this did not reach statistical significance.

Diet and lipolysis in adipose tissue

The expression levels of three proteins related to lipolysis (ATGL, adipose triglyceride lipase; HSL, hormone-sensitive lipase; and $M G L$, monoglyceride lipase) were also examined in AT samples from MetS patients (Fig. 3). We observed an interaction between the quantity and quality of dietary fat and the expression of the ATGL gene $(p<0.001)$. Post hoc Bonferroni's multiple comparison tests showed that $A T G L$ gene expression increased after HSFA diet consumption relative to HMUFA, LFHCC, and LFHCC n-3 diets $(p=0.024, p=0.011$, and $p<0.001$, respectively). No statistically significant differences were observed in $H S L$ and $M G L$ mRNA levels after consumption of any of the four diets tested.

In the postprandial state, we found a significant postprandial increase in the mRNA levels of $A T G L$ after consumption of the HSFA meal $(p<0.001)$ relative to HMUFA $(p=0.004)$ and both LFHCC meals $(p<0.001)$. Additionally, an effect of the quantity and quality of dietary fat on the postprandial HSL mRNA levels was also observed $(p=0.022)$. Post hoc statistical analysis showed a HSL mRNA levels decrease after the intake of the LFHCC meal relative to the HMUFA meal intake $(p=0.031)$. No significant changes were observed in $M G L$ mRNA levels in the postprandial state as compared to the fasting state, for any of the four types of meals analyzed.

Relationships between the expression of lipid metabolism-related genes in adipose tissue with inflammatory markers and body mass index (BMI)

We analyzed the relationship between the expression of the genes related to lipid metabolism and inflammatory markers. A positive correlation between ATGL mRNA levels and the plasma concentration of TNF- $\alpha$ was observed after the dietary intervention in both fasting and postprandial states $(r: 0.440, p=0.006, r: 0.438$, $p=0.006$, respectively).

Finally, we also analyzed the relationship between the expression of the genes studied here and BMI. We found a positive correlation between ATGL mRNA levels in the fasting state after the dietary intervention period and BMI $(r: 0.341, p=0.036)$. 


\section{Discussion}

This study showed that the quantity and quality of the dietary fat differentially modulate the expression of lipogenesis and lipolysis-related factors in the AT of patients with MetS. The long-term consumption of the LFHCC diet enhanced the fasting expression of the lipogenic genes SREBP1 and $S C D 1$, whereas the supplementation of this diet with n-3 PUFA prevented this effect. Additionally, we also observed that the consumption of the HSFA diet increased the expression of the lipolytic gene $A T G L$, relative to the other diets, in both fasting and postprandial states.

Our results suggest that long-term consumption of the two high-fat diets, HSFA and HMUFA, did not alter the lipogenic activity of adipocytes, inasmuch as the expression of neither SREBPI nor SCDI was modified by these diets. Taking into account the fact that lipogenesis is a process that leads to lipid synthesis (lipogenesis "de novo,") our results suggest that the consumption of highfat diets such as HSFA and HMUFA diets do not activate the "de novo" synthesis of lipids in MetS patients. This is in contrast to what was observed for the consumption of a low-fat high-complex carbohydrate diet, which seems to activate lipogenesis in adipocytes, as previously reported in a healthy, overweight population (Minehira et al. 2004).

Interestingly, administration of n-3 FA prevented the stimulatory effect caused by the LFHCC diet on the expression of SREBPI and SCDI genes. To the best of our knowledge, our results show for first time in humans the modulatory effect of n-3 PUFA in reducing the expression of lipogenic genes in AT, which has been described previously in studies with animal models (Ikeda et al. 1998; Muhlhausler et al. 2010).

$S R E B P 1$ is a transcription factor required for the expression of genes related to fatty acid, triglyceride, and phospholipid (Foufelle and Ferre 2002; Hagen et al. 2010). In addition, $S R E B P 1$ also acts as an allostatic mediator that induces adaptive responses to maintain optimal membrane lipid composition in adipocytes (Hagen et al. 2010), and regulates desaturase gene expression (Eberle et al. 2004). Indeed, fatty acids appear to regulate SREBPI activation in a feedback loop, controlling the ratio of unsaturated and saturated fatty acids. To be more specific, transcription and processing of SREBPI is negatively regulated by MUFA and PUFA. In this scenario, our results indicate that the consumption of a low-fat high-complex carbohydrate diet increases the synthesis of lipids in adipose tissue, which would be suppressed through the inhibitory effect induced by n-3 FA on SREBPl expression, probably as a means of preserving membrane fluidity (Hagen et al. 2010).

This idea is supported by the fact that the overexpression of mature SREBP1 overrides the PUFA suppression of lipogenic gene expression (Yahagi et al. 1999), and by a study using animal models which showed that the decrease of the mature form of SREBPI after administration of fish oil rich in $n-3$ PUFA results in decreased expression of lipogenic enzymes including SCDI (Xu et al. 1999). Moreover, n-3 PUFA has been related to the control of the proteolytic processing of SREBP precursors (Pegorier et al. 2004) and its modulator effect in decreasing the lipogenesis process through inhibition of SREBP1 in adipocytes (Madsen et al. 2005).

Our study also showed that the mRNA levels of three desaturases encoding genes increased (FADS3) or tended to increase (DEGS1, and DEGS2) in the postprandial state more after the intake of a HSFA meal relative to the intake of the other three meals, suggesting that the consumption of saturated fat activates the programme to increase the unsaturation of the cellular fatty acids in order to maintain lipid homeostasis and a proper cellular membrane fluidity. Additionally, DEGS1 and DEGS2 genes are involved in the biosynthesis of ceramides/sphingolipids. It has been proposed that this phospholipid impairs insulin signal transduction through the inhibition of Akt/PKB phosphorylation and activation (Stratford et al. 2004), a finding which supports the idea that the consumption of saturated fat impairs insulin sensitivity (Lopez-Miranda et al. 2007).

Moreover, in the postprandial state, we observed a relative decrease the expression of SCD1 after the intake of a HMUFA meal, as compared to the increased observed after the intake of a LFHCC n-3 meal. SCDl is the rate-limiting enzyme in the synthesis of monounsaturated fatty acids and it catalyzes the delta 9-cis desaturation of mainly stearoylCoA to oleoyl-CoA (Zhang et al. 2012), which is further employed for the synthesis of triglycerides and membrane phospholipids (Miyazaki and Ntambi 2003). Our results suggest that the intake of a large amount of the end product of this enzyme, the oleic acid administered in the MUFA meal, decrease $S C D 1$ expression, and thus lipogenesis, by a mechanism that seems to be independent of SREBP1, as no gene expression changes in this gene were observed in this gene in the postprandial state.

The lipolytic activity of adipocytes is also modulated by dietary fat. In response to energy demands, as occurs in the fasting state, lipolysis is activated to release stored fat to provide an energy supply to other tissues (Horowitz 2003). In this report, we show an increase in the expression of ATGL gene in adipose tissue, which encodes for the enzyme involved in the first and rate-limiting steps of lipolysis (Zimmermann et al. 2004), in both, fasting and postprandial state conditions, after the long-term consumption of a HSFA diet relative to the other diets.

Thus, the lipolytic activity of adipocytes rapidly decreases after a fatty meal, due to the inhibitory action exerted by insulin on intracellular lipases, until the end of the postprandial period (Fielding 2011). The observation 
that the expression of the enzyme involved in the first, ratelimiting step of lipolysis, ATGL, increased after the intake of the HSFA meal relative to the other meals suggests that saturated FA causes a refractory state which reduces insulin action on lipolysis. Taking also into account the higher $A T G L$ gene expression values observed in the fasting state after the long-term consumption of HSFA diet relative to the other diets, our results point toward an increased process of lipolysis caused by saturated fat consumption through impaired insulin action (Fielding 2011). The fact that we did not observe any statistically significant difference in plasma non-esterified fatty acid (NEFA) may be explained on the basis that the reduction in insulin action may also be occurring in muscle and liver, and the use of fat instead of glucose after the meal intake by these tissues may compensate the increased release of NEFA by adipose tissue in the postprandial state after saturated fat consumption.

However, a recent study showed no dietary fat effect in the expression of ATGL gene in the adipose tissue of a group of metabolic syndrome patients (van Hees et al. 2012). Nevertheless, the mismatch between this study and the current work may be due to the higher BMI, glucose levels and HOMA index of our metabolic syndrome patients group, which suggest a stronger effect of the saturated fat consumption in the $A T G L$ overexpression in condition of higher insulin resistance, as occurs in our metabolic syndrome patients group. Additionally, other baseline parameters such as TC, TG, c-LDL and c-HDL are slightly higher in our metabolic syndrome patients group.

Moreover, we also found a positive correlation between adipose tissue expression of $A T G L$ and plasma TNF- $\alpha$ levels in both fasting and postprandial state conditions. This is consistent with in vitro data in adipocytes, showing that TNF- $\alpha$ induces lipolysis (Yang et al. 2011) and reinforces the association between inflammation and increased lipolysis, as has previously been shown (Langin et al. 2005). Taken together, these data together with ours suggest that the increase in lipolysis after the consumption of saturated fat may be a consequence of a decrease in insulin sensitivity caused by the inflammatory status, which has indeed been shown to be made worse by saturated fat (Peairs et al. 2011; van Dijk et al. 2009).

Our study also showed a positive relationship between the fasting $A T G L$ gene expression at post-intervention and the BMI of the patients, which agree with a previous study in visceral AT showing that ATGL mRNA levels are higher in morbidly obese patients than in lean subjects (De Naeyer et al. 2011). Taking into account the fact that obese people have low-grade inflammation (Heilbronn and Campbell 2008), the increased expression of $A T G L$ in obese people may be due to the increased inflammation, and the reduction in ATGL mRNA levels when obese people lose weight (Jocken et al. 2007; Viguerie et al. 2005) may be explained by a reduction or disappearance of the low-grade inflammation because of the reduction in body weight. These observations, together with the positive correlation found between $A T G L$ mRNA and plasma TNF- $\alpha$ levels, support the idea of inflammation as the agent responsible for $A T G L$ dysregulation.

One limitation of this study is the reduced sample size, although it is large enough to detect relative changes between diets. Another is the fact that we did not analyze the effect of diet in men and women separately, and our results correspond to men and women together-although these are balanced in the groups. Moreover, small differences in genes expressed with high inter-individual variability may not have been detected, or we may not have had a sufficient sample size to detect small differences in gene expression between groups. Further investigations are needed to extend the knowledge about the quantity and quality of dietary fat in the lipid metabolism and their specific cellular and molecular mechanisms, and to shed more light on nutritionally based therapeutic strategies for the metabolic syndrome.

In conclusion, our results demonstrated the anti-lipogenic effect exerted by LC n-3 PUFA when administered together with a low-fat high-complex carbohydrate diet. In addition, our study also indicated that the consumption of saturated fat increased the expression of the lipolytic gene ATGL relative to the other diets.

Acknowledgments The CIBEROBN is an initiative of the Instituto de Salud Carlos III, Madrid, Spain. This work was supported in part by research grants from the European Union [LIPGENE European Integrated Project-505944], from the Ministerio de Ciencia e Innovacion [grant numbers AGL2004-07907, AGL2006-01979, AGL2009-12270 (to J.L.-M.)]; CIBER Fisiopatologia de la Obesidad y Nutricion [Grant number CB06/03/0047]; Consejeria de Innovacion, Ciencia y Empresa, Junta de Andalucia [Grant number P06-CTS-01425 (to J.L.-M.) CTS-03039 (to MMM)]; and Consejeria de Salud, Junta de Andalucia [Grant numbers 06/128, 07/43, PI-0193 (to J.L.-M.)].); Fondo Europeo de Desarrollo Regional (FEDER). MEM has a fellowship from the Consejo Nacional de Ciencia y Tecnologia, México. (CONACYT). We would like to thank $\mathrm{M}^{\mathrm{a}}$ Jose Gomez-Luna for her technical support. Author contributions: AC, MEM, PP-M, JD-L, FF, FP-J and JLM designed research; AC, MEM, OAR-Z, CM, YA, EMY-S and LG-G conducted research; AC, MEM, PP-M, JD-L, FF, analyzed data; AC, FJT, HMR, MMM, FP-J and JL-M wrote the paper; AC, FP-J and JL$\mathrm{M}$ had primary responsibility for the final content.

Conflict of interest None of the authors has any conflict of interests that could affect the performance of the work or the interpretation of the data.

\section{References}

Breen P, Joseph N, Thompson K et al (2013) Dihydroceramide desaturase knockdown impacts sphingolipids and apoptosis after photodamage in human head and neck squamous carcinoma cells. Anticancer Res 33:77-84 
Cifuentes M, Albala C, Rojas CV (2008) Differences in lipogenesis and lipolysis in obese and non-obese adult human adipocytes. Biol Res 41:197-204

De Naeyer H, Ouwens DM, Van Nieuwenhove Y et al (2011) Combined gene and protein expression of hormone-sensitive lipase and adipose triglyceride lipase, mitochondrial content, and adipocyte size in subcutaneous and visceral adipose tissue of morbidly obese men. Obes Facts 4:407-416

Eberle D, Hegarty B, Bossard P, Ferre P, Foufelle F (2004) SREBP transcription factors: master regulators of lipid homeostasis. Biochimie 86:839-848

Fielding B (2011) Tracing the fate of dietary fatty acids: metabolic studies of postprandial lipaemia in human subjects. Proc Nutr Soc 70:342-350

Foufelle F, Ferre P (2002) New perspectives in the regulation of hepatic glycolytic and lipogenic genes by insulin and glucose: a role for the transcription factor sterol regulatory element binding protein-1c. Biochem J 366:377-391

Grundy SM, Brewer HB Jr, Cleeman JI, Smith SC Jr, Lenfant C (2004) Definition of metabolic syndrome: report of the National Heart, Lung, and Blood Institute/American Heart Association conference on scientific issues related to definition. Arterioscler Thromb Vasc Biol 24:e13-e18

Hagen RM, Rodriguez-Cuenca S, Vidal-Puig A (2010) An allostatic control of membrane lipid composition by SREBP1. FEBS Lett 584:2689-2698

Havel PJ (2002) Control of energy homeostasis and insulin action by adipocyte hormones: leptin, acylation stimulating protein, and adiponectin. Curr Opin Lipidol 13:51-59

Heilbronn LK, Campbell LV (2008) Adipose tissue macrophages, low grade inflammation and insulin resistance in human obesity. Curr Pharm Des 14:1225-1230

Horowitz JF (2003) Fatty acid mobilization from adipose tissue during exercise. Trends Endocrinol Metab 14:386-392

Ikeda I, Cha JY, Yanagita T et al (1998) Effects of dietary alphalinolenic, eicosapentaenoic and docosahexaenoic acids on hepatic lipogenesis and beta-oxidation in rats. Biosci Biotechnol Biochem 62:675-680

Jocken JW, Langin D, Smit E et al (2007) Adipose triglyceride lipase and hormone-sensitive lipase protein expression is decreased in the obese insulin-resistant state. J Clin Endocrinol Metab 92:2292-2299

Khoury DE, Hwalla N, Frochot V, Lacorte JM, Chabert M, Kalopissis AD (2009) Postprandial metabolic and hormonal responses of obese dyslipidemic subjects with metabolic syndrome to test meals, rich in carbohydrate, fat or protein. Atherosclerosis 210:307-313

Langin D, Dicker A, Tavernier G et al (2005) Adipocyte lipases and defect of lipolysis in human obesity. Diabetes 54:3190-3197

Lopez-Miranda J, Perez-Martinez P, Marin C, Fuentes F, Delgado J, Perez-Jimenez F (2007) Dietary fat, genes and insulin sensitivity. J Mol Med 85:213-226

Madsen L, Petersen RK, Kristiansen K (2005) Regulation of adipocyte differentiation and function by polyunsaturated fatty acids. Biochim Biophys Acta 1740:266-286

Meneses ME, Camargo A, Perez-Martinez P et al (2011) Postprandial inflammatory response in adipose tissue of patients with metabolic syndrome after the intake of different dietary models. Mol Nutr Food Res 55:1759-1770

Minehira K, Vega N, Vidal H, Acheson K, Tappy L (2004) Effect of carbohydrate overfeeding on whole body macronutrient metabolism and expression of lipogenic enzymes in adipose tissue of lean and overweight humans. Int J Obes Relat Metab Disord 28:1291-1298

Miyazaki M, Ntambi JM (2003) Role of stearoyl-coenzyme A desaturase in lipid metabolism. Prostaglandins Leukot Essent Fatty Acids 68:113-121
Muhlhausler BS, Cook-Johnson R, James M, Miljkovic D, Duthoit E, Gibson R (2010) Opposing effects of omega-3 and omega-6 long chain polyunsaturated Fatty acids on the expression of lipogenic genes in omental and retroperitoneal adipose depots in the rat. J Nutr Metab 2010:1-9, Article id 927836. doi:10.1155/2010/ 927836

Peairs AD, Rankin JW, Lee YW (2011) Effects of acute ingestion of different fats on oxidative stress and inflammation in overweight and obese adults. Nutr J 10:122

Pedrono F, Blanchard H, Kloareg $\mathrm{M}$ et al (2010) The fatty acid desaturase 3 gene encodes for different FADS3 protein isoforms in mammalian tissues. J Lipid Res 51:472-479

Pegorier JP, Le May C, Girard J (2004) Control of gene expression by fatty acids. J Nutr 134:2444S-2449S

Schmitz-Peiffer C (2000) Signalling aspects of insulin resistance in skeletal muscle: mechanisms induced by lipid oversupply. Cell Signal 12:583-594

Shaw DI, Tierney AC, McCarthy S et al (2009) LIPGENE foodexchange model for alteration of dietary fat quantity and quality in free-living participants from eight European countries. Br J Nutr 101:750-759

Shimomura I, Matsuda M, Hammer RE, Bashmakov Y, Brown MS, Goldstein JL (2000) Decreased IRS-2 and increased SREBP-1c lead to mixed insulin resistance and sensitivity in livers of lipodystrophic and ob/ob mice. Mol Cell 6:77-86

Stratford S, Hoehn KL, Liu F, Summers SA (2004) Regulation of insulin action by ceramide: dual mechanisms linking ceramide accumulation to the inhibition of Akt/protein kinase B. J Biol Chem 279:36608-36615

Suganami T, Tanaka M, Ogawa Y (2012) Adipose tissue inflammation and ectopic lipid accumulation [Review]. Endocr J 59:849-857

Sun K, Kusminski CM, Scherer PE (2011) Adipose tissue remodeling and obesity. J Clin Invest 121:2094-2101

Ternes P, Franke S, Zahringer U, Sperling P, Heinz E (2002) Identification and characterization of a sphingolipid delta 4-desaturase family. J Biol Chem 277:25512-25518

van Dijk SJ, Feskens EJ, Bos MB et al (2009) A saturated fatty acidrich diet induces an obesity-linked proinflammatory gene expression profile in adipose tissue of subjects at risk of metabolic syndrome. Am J Clin Nutr 90:1656-1664

van Hees AM, Jocken JW, Essers Y, Roche HM, Saris WH, Blaak EE (2012) Adipose triglyceride lipase and hormone-sensitive lipase protein expression in subcutaneous adipose tissue is decreased after an isoenergetic low-fat high-complex carbohydrate diet in the metabolic syndrome. Metabolism 61:1404-1412

Viguerie N, Vidal H, Arner P et al (2005) Adipose tissue gene expression in obese subjects during low-fat and high-fat hypocaloric diets. Diabetologia 48:123-131

Weyer C, Foley JE, Bogardus C, Tataranni PA, Pratley RE (2000) Enlarged subcutaneous abdominal adipocyte size, but not obesity itself, predicts type II diabetes independent of insulin resistance. Diabetologia 43:1498-1506

Xu J, Nakamura MT, Cho HP, Clarke SD (1999) Sterol regulatory element binding protein-1 expression is suppressed by dietary polyunsaturated fatty acids. A mechanism for the coordinate suppression of lipogenic genes by polyunsaturated fats. J Biol Chem 274:23577-23583

Yahagi N, Shimano H, Hasty AH et al (1999) A crucial role of sterol regulatory element-binding protein- 1 in the regulation of lipogenic gene expression by polyunsaturated fatty acids. J Biol Chem 274:35840-35844

Yang X, Zhang X, Heckmann BL, Lu X, Liu J (2011) Relative contribution of adipose triglyceride lipase and hormone-sensitive lipase to tumor necrosis factor-alpha (TNF-alpha)-induced lipolysis in adipocytes. J Biol Chem 286:40477-40485 
Zhang J, Kris-Etherton PM, Thompson JT, Hannon DB, Gillies PJ, Vanden Heuvel JP (2012) Alpha-linolenic acid increases cholesterol efflux in macrophage-derived foam cells by decreasing stearoyl CoA desaturase 1 expression: evidence for a farnesoidX-receptor mechanism of action. J Nutr Biochem 23:400-409
Zimmermann R, Strauss JG, Haemmerle G et al (2004) Fat mobilization in adipose tissue is promoted by adipose triglyceride lipase. Science 306:1383-1386 